\title{
Association between Peripheral Leukocyte Telomere Length and Dietary Vitamin $A$ and Carotenoid in U.S. Adults
}

\author{
Li Yi ${ }^{1}$, Gao Lijie', Han Nannan², Wang Chaoqun ${ }^{3}$, Luo Weijia ${ }^{4}$, Tian Ye ${ }^{1,2, *}$, Shi Wenzhen ${ }^{2, "}$ \\ ${ }^{1}$ Medicine college, Yan'an University, Yan'an, PR China \\ ${ }^{2}$ Neurology Department, Xi'an No.3 hospital, Xi'an, PR China \\ ${ }^{3}$ Respiratory Department, Chengdu Second Hospital of Traditional Chinese Medicine, Chengdu, PR China \\ ${ }^{4}$ Respiratory Department, MeiShan Hospital of Traditional Chinese Medicine, MeiShan, PR China \\ *Corresponding author: shiwenzhen736@163.com, chhty@sina.com
}

Received February 07, 2019; Revised March 18, 2019; Accepted April 03, 2019

\begin{abstract}
To investigate the independent association between peripheral leukocyte telomere length and dietary vitamin A and carotenoid, we collected 839 participants older than 20-year from the 1999-2000 National Health and Nutrition Examination Survey. The peripheral leukocyte telomere length was obtained using the quantitative polymerase chain reaction method. Dietary information, such as alcohol, caffeine, carotenoid, folate and vitamin $\mathrm{A} / \mathrm{B} 1 / \mathrm{B} 2 / \mathrm{B} 6 / \mathrm{B} 12 / \mathrm{C} / \mathrm{E}$ was from a recall of the previous $24 \mathrm{~h}$ taken food. Multivariable logistic regression and smooth curve fitting was conducted to analyze the independent association between telomere length and dietary vitamin A and carotenoid. Obesity was further treated as effect modification factor in the stratified analysis. After adjusting for age, race, sex, education, obesity, physical activity, PIR, smoking status, CAD history, serum concentrations of vitamin E/A/B12, folate, gamma-tocopherol, CRP, total cholesterol, cadmium and dietary intake of alcohol, caffeine, fiber, folate and vitamin B1/B2/B6/B12/C/E, the daily vitamin A and carotenoid intake was not significantly associated with telomere length in general population (vitamin A: $0.0009,95 \%$ CI: $-0.0041,0.0058$; carotenoid: $0.0040,95 \%$ CI: $-0.0458,0.0538$ ). In non-obesity population, the association coefficient was -0.0121 (95\% CI: $-0.0259,0.0017)$ for vitamin A and -0.1369 (95\% CI: $-0.2809,0.0070)$ for carotenoid. Vitamin A and carotenoid consumption was not associated with peripheral leukocyte telomere length independently in general adult population. However, in non-obese individuals, increasing vitamin A and carotenoid might tend to associate with decreasing peripheral leukocyte telomere length.
\end{abstract}

Keywords: diet nutrition, antioxidative nutrient, aging, obesity

Cite This Article: Li Yi, Gao Lijie, Han Nannan, Wang Chaoqun, Luo Weijia, Tian Ye, and Shi Wenzhen, "Association between Peripheral Leukocyte Telomere Length and Dietary Vitamin A and Carotenoid in U.S. Adults." Journal of Food and Nutrition Research, vol. 7, no. 4 (2019): 279-286. doi: 10.12691/jfnr-7-4-4.

\section{Introduction}

Locating at both ends of chromosomes, telomere contains a repetitive sequence of 5'-TTAGGGn-3' serving to protect chromosome stability and integrity, adjust cellular proliferation, and prevent chromosome end fusions [1,2]. Telomere length would be shortened due to failing replication of partial telomeric DNA sequence in cell division $[3,4]$. Thus, telomere length has become one of biological aging markers to monitor chronic diseases risks $[5,6,7,8]$ and potential death risk $[8,9,10]$. A variety of factors could increase (physical activity $[11,12,13]$, and normal BMI $[12,13,14]$ etc.) or decrease (smoking [11], alcohol [11], and mental stress [15] etc.) telomere length. AS age increases, telomeres become shorter by about 15 base pairs per year $[16,17]$.
Diet can also affect the telomere length $[18,19,20]$. Some nutrients can increase the telomere length, while others may shorten it. For instance, individuals who ate high levels of nuts and seeds tended to have longer telomeres than those who consumed less [21]. Fruit and vegetable intake were also suggested positively associated with telomere length $[19,20,22,23,24]$. Other foods might have the reverse influence. The processed meat, sweetened carbonated beverage and sugar-sweetened soda intake might shorten telomere length $[19,25,26]$.

The underlying pathway leading to telomere length change might be oxidative reaction and inflammation $[27,28]$. There is evidence indicating that the intake of antioxidants and DNA-repair vitamins may affect telomeres length $[18,29,30]$. Dietary vitamin A was a basic nutrient important in growth, immunity vision and cell division [31]. Vitamin A might influence peripheral telomere length through the potential mechanisms of gene expression 
and epigenetic modifications regulation and immune function [32-37]. Carotenoids, converting to retinol and then involving to the vitamin A metabolism, are positively correlated to aging related diseases via antioxidative effects $[38,39,40]$. Therefore, vitamin A and carotenoid might exert protective effect on telomere length by antioxidation and antiinflammation mechanism.

Serum vitamin A [41] and carotenoids [42] have been reported positively related to telomere length. Up to date, there is no investigation of the association between dietary vitamin A and carotenoid with telomere length. Although recent study suggested dietary pattern which enrich in vitamin tended to associate with longer telomere length [43], the independent association between each dietary vitamin and telomere length is rather not clear.

The NHANSE was a cross-sectional designed nationally survey targeting at providing trustworthy diseases related information. The surveying sample has a national representativeness. And the standard protocol assured the quality of data collection. In this study, we aimed to address the association between the peripheral leukocyte telomere length and dietary vitamin A as well as carotenoid, according to the 1999-2000 NHANES in United States adult population.

\section{Materials and Methods}

\subsection{Data Collection}

NHANSE is conducted by the National Center for Health Statistics to measure the health and nutritional condition of non-institutionalized citizens lived in United State. This program has become continuous since 1999. However, the survey contents of this program might vary with different health focus in every two years. All participants are chosen from a multistage cluster design method to guarantee the sample representativeness. Written consents had been obtained from participants for the data use. The survey data were publicly available to download (http://www.cdc.gov/nchs/nhanes/nhanes questionnaires.h tm). The corresponding variants codebook provided by NHANSE program could also be publicly accessed (https://wwwn.cdc.gov/nchs/nhanes/search/variablelist.asp $\mathrm{x})$. The data file compromised demographic characteristics, physical exam results, laboratory results, and questionnaire items. The analyzed data in this study were from 1999-2000 cycle [44].

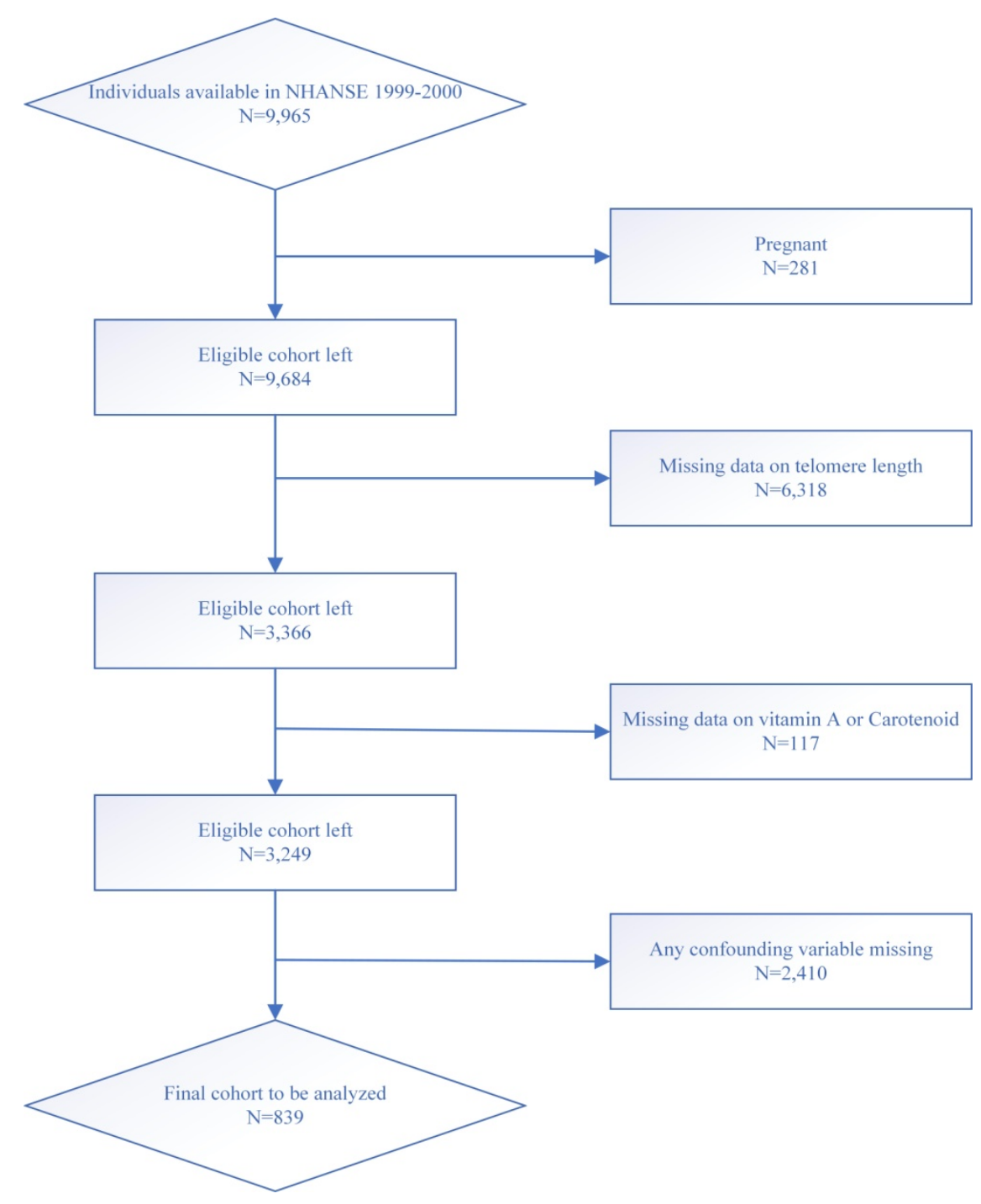

Figure 1. Eligible individuals in the exploration of the associations between leucocyte telomere length and dietary vitamin A and Carotene in adults. Confounding variables including: age, race, sex, education, obesity, physical activity, PIR, smoking status, CAD history, serum concentrations of vitamin E/A/B12, folate, gamma-tocopherol, CRP, total cholesterol, cadmium as well as dietary intake of alcohol, caffeine, fiber, folate and dietary vitamin $\mathrm{A} / \mathrm{C} / \mathrm{E} / \mathrm{B} 1 / \mathrm{B} 2 / \mathrm{B} 6 / \mathrm{B} 12$ 
The 1999-2000 NHANSE dataset comprised 9,965 participants. Since the recommended daily intake of vitamin $\mathrm{A}$ and carotenoid differed in pregnant and non-pregnant population, we excluded the 281 participants in 1999-2000 cycle. DNA samples for telomere length examination were acquired from participants older than 20 -year-old. Thus, we directly eliminated the telomere length missing participants without age filtering, remaining 3,366 eligible participants. The vitamin A and carotenoid screening abandoned 117 participants. Further 2,410 participants were removed for the missing of possible confounding variants, including age, race, sex, education, obesity, physical activity, PIR, smoking status, history of CAD, serum concentrations of vitamin E/A/B12, folate, gamma-tocopherol, CRP, total cholesterol, cadmium as well as dietary intake of alcohol, caffeine, fiber, and dietary vitamin $\mathrm{A} / \mathrm{C} / \mathrm{E} / \mathrm{B} 1 / \mathrm{B} 2 / \mathrm{B} 6 / \mathrm{B} 12$ and folate. The final cohort to be analyzed remained 839 participants, as shown in Figure 1.

\subsection{Peripheral Leukocyte Telomere Length}

With the Puregene (D-50K) kit protocol (Gentra Systems, Inc., Minneapolis, Minnesota), DNA samples were obtained from peripheral leukocyte in the whole blood. Then, in the Dr. Elizabeth Blackburn laboratory at the University of California, San Francisco, the quantitative polymerase chain reaction method served to measure the telomere length relative to standard reference DNA (T/S ratio). The detailed method was reported by Cawthon [45] and Lin et al. [46] previously. Each DNA sample was tested twice at three different timepoint. As recorded by NHANSE, "Sample plates were assayed in groups of 3 plates, and no 2 plates were grouped together more than once. Each assay plate contained 96 control wells with 8 control DNA samples. Assay runs with 8 or more invalid control wells were excluded from further analysis ( $<1 \%$ of runs). Control DNA values were used to normalize between-run variability. Runs with more than 4 control DNA values falling outside 2.5 standard deviations from the mean for all assay runs were excluded from further analysis ( $<6 \%$ of runs). For each sample, any potential outliers were identified and excluded from the calculations $(<2 \%$ of samples). The mean and standard deviation of the $\mathrm{T} / \mathrm{S}$ ratio were then calculated normally. The intraassay coefficient of variation was $6.5 \%$." We converted the peripheral leukocyte telomere length from the original $\mathrm{T} / \mathrm{S}$ ratio to base pairs $(\mathrm{bp})$ with the formula $3,274+2,413 *(\mathrm{~T} / \mathrm{S})[47]$.

\subsection{Dietary Vitamin A and Carotenoid}

The dietary information was from a $24 \mathrm{~h}$ (midnight to midnight) recall. Bilingual interviewers were demanded to have a Bachelor's Degree in Food and Nutrition or Home Economics with at least 10 credit hours in food and nutrition. And they all finished a two-week training program to improve interviewing quality. All participants of the 1999-2000 survey were able to have the dietary interview. Interviewees were randomly assigned to two interviewing group. Half of the them completed the dietary recall face-to-face, another by telephone. Interviewers then follow the operations of a computer-assisted dietary interview system, which provided a standardized format to collect detailed food and beverage information. A postrecall questionnaire was conducted after the 24-hour recall. The total dietary intake of vitamin A and carotenoid was calculated from the recall data [48].

\subsection{Confounding Variables}

Demographic characteristics, including age, race, sex, education, obesity, physical activity, PIR, smoking status and $\mathrm{CAD}$, were considered into statistical adjustment. Race covered the non-Hispanic white, non-Hispanic black, Mexican, Hispanic white, and other races including multiraces resident in United Stated. Education was clarified into three parts: less than high school, high school, and more than a high school. PIR was categorized into low, moderate and high group based on the mean value. Obesity was defined as body mass index $(\mathrm{BMI}) \geq 25 \mathrm{~kg} / \mathrm{m}^{2}$. The intensity of physical activity was ranked into three grades referring to mild, moderate and vigorous physical activity according to MET. MET $<3$ was classified to mild grade; $\mathrm{MET}=3$ or $\mathrm{MET}<6$ was classified to moderate grade; and MET $=6$ or MET $>6$ was classified to vigorous grade. Smoking status described whether participants were currently smoking or not. The history of CAD was from self-report through questionnaire [49].

Serum concentrations of vitamin A/E/B12, folate, gamma tocopherol, cadmium, total cholesterol and CRP were also brought into adjustment. The detailed method of laboratory tests could be obtained publicly online [50]. Besides, total dietary intake of alcohol, caffeine, fiber, folate, and vitamin A/C/E/B1/B2/B6/B12 were involved into the potential confounding variable. Dietary covariant were obtained from dietary questionnaire as mentioned before [48].

\subsection{Statistical Analysis}

In conformity with NHANSE analytical guidelines [51], 2-year weights of subsample were applied to statistical analyses. All analyses were carried out with Empower Stats (http://www.empowerstats.com) and the R software, version 3.3.1 (http://www.R-project.org/). The two-sided $\mathrm{P}<0.05$ was considered to be statistically significant. Data were expressed as mean \pm standard error (SD) for continuous variables and frequency (\%) for categorical variables in male and female population. The difference in gender subgroup was performed using Independent Student's t-tests or Mann-Whitney U-tests. Multiple regression model was used to detect the independent association between peripheral leukocyte length and $24 \mathrm{~h}$ total intake of vitamin A and carotenoid. The smooth curve fitting was employed to briefly present the correlation. Stratification analysis was also performed to address the modification effect of covariant.

\section{Results and Discussion}

\subsection{Clinical Characteristics}

The total size of participants analyzed in this investigation was 839 (Figure 1). Clinical characteristics in different gender were displayed in Table 1 . The average 
age was $53.5 \pm 17.7$ in male, and $51.8 \pm 16.6$ in female. Significant difference was not found in mean peripheral leukocyte telomere length $(P=0.82)$ among males and females. The average telomere length in men was 5610.6 \pm $605.7 \mathrm{bp}$ and $5620.2 \pm 550.2 \mathrm{bp}$ in women. The distribution of race, education, smoking status, and CAD history was significantly different between the gender group. The average concentration of serum vitamin A was $62.8 \pm 17.4$ $\mathrm{ug} / \mathrm{dL}$ in male, while in female it is significantly lower at $57.5 \pm 16.7 \mathrm{ug} / \mathrm{dL}(P<0.01)$. Serum CRP level was also significantly different among two genders with $0.4 \pm 0.8 \mathrm{mg} / \mathrm{dL}$ in male and $0.7 \pm 1.8 \mathrm{mg} / \mathrm{dL}(P<0.01)$. Concentrations of other circulating nutrients and cadmium did not statistically differ in males and females.

Table 1. Clinical characteristic of the study population from NHANSE 1999-2000

\begin{tabular}{|c|c|c|c|}
\hline Sex & Male $(\mathrm{N}=509)$ & Female $(\mathrm{N}=330)$ & $P$ \\
\hline Age & $53.5 \pm 17.7$ & $51.8 \pm 16.6$ & 0.18 \\
\hline Ethnicity ${ }^{1}$ & & & 0.05 \\
\hline 1 & $263(51.7 \%)$ & $186(56.4 \%)$ & \\
\hline 2 & $60(11.8 \%)$ & $50(15.2 \%)$ & \\
\hline 3 & $143(28.1 \%)$ & $70(21.2 \%)$ & \\
\hline 4 & $25(4.9 \%)$ & $19(5.8 \%)$ & \\
\hline 5 & $18(3.5 \%)$ & $5(1.5 \%)$ & \\
\hline Education $^{2}$ & & & 0.03 \\
\hline 1 & $211(41.5 \%)$ & $107(32.4 \%)$ & \\
\hline 2 & $122(24.0 \%)$ & $90(27.3 \%)$ & \\
\hline 3 & $176(34.6 \%)$ & $133(40.3 \%)$ & \\
\hline PIR & & & 0.63 \\
\hline Low & $120(23.6 \%)$ & $87(26.4 \%)$ & \\
\hline Middle & $168(33.0 \%)$ & $102(30.9 \%)$ & \\
\hline High & $221(43.4 \%)$ & $141(42.7 \%)$ & \\
\hline Smoking & & & $<0.01$ \\
\hline 0 & $350(68.8 \%)$ & $196(59.4 \%)$ & \\
\hline 1 & $159(31.2 \%)$ & $134(40.6 \%)$ & \\
\hline Physical Activity & & & 0.37 \\
\hline Mild & $13(2.6 \%)$ & $4(1.2 \%)$ & \\
\hline Moderate & $279(54.8 \%)$ & $188(57.0 \%)$ & \\
\hline Vigorous & $217(42.6 \%)$ & $138(41.8 \%)$ & \\
\hline Obesity & & & 0.20 \\
\hline 0 & $147(28.9 \%)$ & $109(33.0 \%)$ & \\
\hline 1 & $362(71.1 \%)$ & $221(67.0 \%)$ & \\
\hline $\mathrm{CAD}^{3}$ & & & $<0.01$ \\
\hline 0 & $476(93.5 \%)$ & $321(97.3 \%)$ & \\
\hline 1 & $31(6.1 \%)$ & $6(1.8 \%)$ & \\
\hline 2 & $2(0.4 \%)$ & $3(0.9 \%)$ & \\
\hline \multicolumn{4}{|l|}{ Laboratory tests } \\
\hline Cadmium (ug/L) & $0.8 \pm 0.6$ & $0.9 \pm 0.6$ & 0.02 \\
\hline Vitamin A (ug/dL) & $62.8 \pm 17.4$ & $57.5 \pm 16.7$ & $<0.01$ \\
\hline Folate $(\mathrm{ng} / \mathrm{mL})$ & $14.9 \pm 9.1$ & $15.6 \pm 11.2$ & 0.37 \\
\hline Vitamin B12(pg/mL) & $520.6 \pm 713.2$ & $1603.6 \pm 13731.6$ & 0.08 \\
\hline Gamma tocopherol (ug/dL) & $242.4 \pm 141.4$ & $255.7 \pm 154.1$ & 0.23 \\
\hline Vitamin E (ug/dL) & $1313.8 \pm 580.4$ & $1340.3 \pm 662.0$ & 0.54 \\
\hline $\mathrm{CRP}(\mathrm{mg} / \mathrm{dL})$ & $0.4 \pm 0.8$ & $0.7 \pm 1.8$ & $<0.01$ \\
\hline Total cholesterol (mmol/L) & $5.3 \pm 1.0$ & $5.4 \pm 1.0$ & 0.11 \\
\hline Telomere Length(bp) & $5610.6 \pm 605.7$ & $5620.2 \pm 550.2$ & 0.82 \\
\hline
\end{tabular}

Data are presented as number (percentage) or means \pm standard deviation. ${ }^{1}$ the code of ethnicity represented non-Hispanic white, non-Hispanic black, Mexican American, other Hispanic and other races (including multi races) from 1 to 5 respectively.

${ }^{2}$ the code of education represented less than high school, high school diploma and more than high school from 1 to 3 respectively.

${ }^{3}$ the history of CAD was recorded as non-CAD history $(0)$, CAD history (1) and not sure (2).

\subsection{Daily Dietary Vitamin A and Carotenoid Consumption}

When it comes to the amount of dietary intake in previous $24 \mathrm{~h}$, the total intake of vitamin A $(P=0.62)$ or carotenoid $(P=0.63)$ did not significantly varied in different gender. Mean daily intake of vitamin A was $6140.4 \mathrm{IU}$ in males and 5798.1 IU in females. And the mean daily intake of carotenoid was $434.7 \mathrm{RE}$ in males and $465.9 \mathrm{RE}$ in females. The median daily intake of vitamin A was 3379.6 IU in males and 2809.9 IU in females. For carotenoid, the median daily intake was 173.0 RE in males and 165.5 RE in females. However, in male the vitamin A intake of previous $24 \mathrm{~h}$ spanned from $85.4 \mathrm{IU}$ to $72,195.4 \mathrm{IU}$, as for carotenoid the range was from 0 to $7182.3 \mathrm{RE}$. In female, the range of vitamin A intake was from 0 to $165,440.7 \mathrm{IU}$, and the range of carotenoid was from 0 to 16532.4 RE. Dietary intakes were shown in Table 2.

Table 2. Means, median and range of previous $24 \mathrm{~h}$ total dietary intakes

\begin{tabular}{|c|c|c|c|c|}
\hline & & Male $(\mathrm{N}=509)$ & $\begin{array}{c}\text { Female } \\
(\mathrm{N}=330)\end{array}$ & $P$ \\
\hline \multirow{4}{*}{$\begin{array}{l}\text { Vitamin A } \\
\text { (IU) }\end{array}$} & Mean & 6140.4 & 5798.1 & 0.62 \\
\hline & Median & 3379.6 & 2809.9 & \\
\hline & Range & $85.4-72,195.4$ & $0.0-165,440.7$ & \\
\hline & $>10,000 \mathrm{IU} / \mathrm{d}$ & $73(14.3 \%)$ & $47(14.2 \%)$ & \\
\hline \multirow{3}{*}{$\begin{array}{l}\text { Carotenoid } \\
\text { (RE) }\end{array}$} & Mean & 434.7 & 465.9 & 0.63 \\
\hline & Median & 173.0 & 165.5 & \\
\hline & Range & $0.0-7182.3$ & $0.0-16532.4$ & \\
\hline \multirow{3}{*}{$\begin{array}{l}\text { Vitamin B1 } \\
(\mathrm{mg})\end{array}$} & Mean & 1.8 & 1.3 & $<0.01$ \\
\hline & Median & 1.6 & 1.1 & \\
\hline & Range & $0.0-939.0$ & $0.0-213.1$ & \\
\hline \multirow{3}{*}{$\begin{array}{l}\text { Vitamin B2 } \\
(\mathrm{mg})\end{array}$} & Mean & 2.1 & 1.5 & $<0.01$ \\
\hline & Median & 1.8 & 1.4 & \\
\hline & Range & $0.2-8.6$ & $0.0-7.2$ & \\
\hline \multirow{3}{*}{$\begin{array}{l}\text { Vitamin B6 } \\
(\mathrm{mg})\end{array}$} & Mean & 2.1 & 1.4 & $<0.01$ \\
\hline & Median & 1.8 & 1.2 & \\
\hline & Range & $0.2-7.6$ & $0.1-8.5$ & \\
\hline \multirow{3}{*}{$\begin{array}{l}\text { Total Folate } \\
\text { (mcg) }\end{array}$} & Mean & 413.3 & 294.7 & $<0.01$ \\
\hline & Median & 365.7 & 256.4 & \\
\hline & Range & $22.9-2289.2$ & $20.4-1957.5$ & \\
\hline \multirow{3}{*}{$\begin{array}{l}\text { Vitamin B12 } \\
(\mathrm{mg})\end{array}$} & Mean & 5.9 & 3.6 & $<0.01$ \\
\hline & Median & 4.0 & 2.7 & \\
\hline & Range & $0.0-141.4$ & $0.1-32.3$ & \\
\hline \multirow{3}{*}{$\begin{array}{l}\text { Vitamin } C \\
(\mathrm{mg})\end{array}$} & Mean & 113.4 & 83.4 & $<0.01$ \\
\hline & Median & 71.0 & 55.0 & \\
\hline & Range & $0.1-940.6$ & $0.0-747.6$ & \\
\hline \multirow{3}{*}{$\begin{array}{l}\text { Vitamin } E \\
(\mathrm{mg})\end{array}$} & Mean & 9.1 & 7.5 & $<0.01$ \\
\hline & Median & 7.7 & 6.0 & \\
\hline & Range & $0.5-70.2$ & $0.2-97.0$ & \\
\hline \multirow{3}{*}{ Fiber (gm) } & Mean & 17.9 & 12.2 & $<0.01$ \\
\hline & Median & 15.6 & 10.3 & \\
\hline & Range & $0.0-111.8$ & $0.9-44.0$ & \\
\hline \multirow{3}{*}{ Caffeine (mg) } & Mean & 258.4 & 217.0 & 0.08 \\
\hline & Median & 158.5 & 137.4 & \\
\hline & Range & $0.0-4159.9$ & $0.0-1885.9$ & \\
\hline \multirow{3}{*}{ Alcohol (gm) } & Mean & 18.7 & 8.8 & 0.01 \\
\hline & Median & 0.0 & 0.0 & \\
\hline & Range & $0.0-939.0$ & $0.0-213.1$ & \\
\hline
\end{tabular}

Dietary vitamin A mainly came from animal origin foods in the form of retinol and plants in carotenoid [52,53]. Dairy products and fortified breakfast cereals were also added with vitamin A. Among these 
sources, dairy was the major resource of vitamin A [54]. And beta-carotene was the most notably provitamin A carotenoid in plant foods. In the present investigation, vitamin A was calculated from the recall of previous $24 \mathrm{~h}$ eaten foods, including the retinol and provitamin A carotenoid [48]. NIH recommended daily vitamin A consumption should not be over 10,000 IU per day for adults older than 19-year [54]. The percentage of analyzed participants whose vitamin A consumption exceeded the recommend upper limitation was $14.3 \%$ in men and $14.2 \%$ in women. Therefore, the daily vitamin A intake might be excessive in American residents.

\subsection{Association between Peripheral Leukocyte Telomere Length and Dietary Vitamin A and Carotenoid}

In the crude linear regression model (Table 3.), the previous $24 \mathrm{~h}$ dietary vitamin A intake was not significantly associated with peripheral leukocyte telomere length $(\beta=-0.0005,95 \% \mathrm{CI}:-0.0035,0.0025$, $P=0.75)$, either did carotenoid intake $(\beta=-0.0067$, 95\% CI: $-0.0373,0.0238, P=0.67)$. Then, we adjusted demographic variables, such as age, sex, ethnicity, education, PIR, CAD history, physical activity, obesity and smoking status. The association was not found out (for dietary vitamin A: $\beta=-0.0011,95 \%$ CI: -0.0040 , $0.0018, P=0.47$; for dietary carotenoid: $\beta=-0.0131$, 95\% CI: $-0.0424,0.0162, P=0.38)$. After adjusting demographic and serum laboratory examinations, including total cholesterol, CRP, cadmium, folate, gamma tocopherol and vitamin $\mathrm{A} / \mathrm{E} / \mathrm{B} 12$, the association was still negative (for dietary vitamin A: $\beta=0.0002,95 \% \mathrm{CI}:-0.0043$, $0.0048, P=0.92$; for dietary carotenoid: $\beta=-0.0002,95 \%$ CI: $-0.0477,0.0473, P=0.97)$. We further controlled the dietary related covariant, including alcohol, caffeine, folate and vitamin B1/B2/B6/B12/C/E. The association of dietary telomere length with vitamin A intake was 0.0009 (95\% CI: $-0.0041,0.0058)$ without any significant difference $(P=0.73)$; and with carotenoid was 0.0040 (95\% CI: $-0.0458,0.0538)$ also without any significant difference $(P=0.88)$.

However, our founding was a paradox with other investigations. An Italian cross-sectional study of 56 samples found that higher consumption of vegetables, especially the antioxidant nutrients such as beta-carotene were correlated with longer peripheral leukocyte telomere length [24]. In this study, daily dietary vitamin A was significantly associated with telomere length $(r=0.325$, $P=0.02$ ) by the method of Pearson correlation analysis without any covariant controlled. After adjusting for age, gender, smoking status, food categories and micronutrients, daily beta-carotene intake was also positively associated with telomere length $(\beta=0.178 ; P=0.0004)$. Circulating level of vitamin A and carotenoid was also suggested to be positively associated with peripheral leukocyte telomere length in United States adults according to NHANSE database $[41,42]$.

\subsection{Association between Peripheral Leukocyte Telomere Length and Dietary Vitamin A and Carotenoid Modified by Obesity}

Interaction effect was also analyzed among confounding variables (Table 4). With adjusting age, sex, ethnicity, education, PIR, CAD history, physical activity, smoking status, total cholesterol, CRP, cadmium, folate, gamma tocopherol, vitamin $\mathrm{A} / \mathrm{E} / \mathrm{B} 12$, alcohol consumption as well as dietary caffeine, folate and vitamin $\mathrm{B} 1 / \mathrm{B} 2 / \mathrm{B} 6 / \mathrm{B} 12 / \mathrm{C} / \mathrm{E}$, obesity significantly modified the association of peripheral telomere length and dietary intake of vitamin A $(P=0.02)$ and carotenoid $(P=0.04)$. Among non-obesity individuals, per $1000 \mathrm{IU}$ increase of dietary vitamin A might shorten $12 \mathrm{bp}$ of peripheral leukocyte telomere length (95\% CI: $-25.9,1.7)$; And per 100 RE increase of dietary carotenoid might decrease around $13 \mathrm{bp}$ of peripheral leukocyte telomere length (95\% CI: -28.09, 0.7). In non-obesity populations, the distribution range of coefficient was quiet near the borderline. If measured in base pairs, per $15 \mathrm{bp}$ shortening of telomere length indicated 12-month aging [16,17]. For non-obesity participants, each 1,000 IU increasing of vitamin A consumption associated with around $12 \mathrm{bp}$ decreasing of telomere length, indicating 9.6-month aging. And each $100 \mathrm{RE}$ increasing of carotenoid consumption associated with around $14 \mathrm{bp}$ decreasing of telomere length, indicating 11.2-month aging. Therefore, overtaking vitamin A or carotenoid might not benefit telomere length. Non-obesity individuals might even acquire harmful effect on telomere length with too much intake of vitamin A and carotenoid.

Table 3. Coefficients and $95 \%$ CIs for peripheral leukocyte telomere length(bp) of vitamin A and carotenoid

\begin{tabular}{|c|c|c|c|c|}
\hline & \multicolumn{2}{|l|}{ Vitamin A } & \multicolumn{2}{|l|}{ Carotenoid } \\
\hline & $\beta(95 \% \mathrm{CI})$ & $P$ & $\beta(95 \% \mathrm{CI})$ & $P$ \\
\hline Crude $^{1}$ & $-0.0005(-0.0035,0.0025)$ & 0.75 & $-0.0067(-0.0373,0.0238)$ & 0.67 \\
\hline Model I & $-0.0011(-0.0040,0.0018)$ & 0.47 & $-0.0131(-0.0424,0.0162)$ & 0.38 \\
\hline Model $\mathrm{II}^{3}$ & $0.0002(-0.0043,0.0048)$ & 0.92 & $-0.0002(-0.0477,0.0473)$ & 0.97 \\
\hline Model $\mathrm{III}^{4}$ & $0.0009(-0.0041,0.0058)$ & 0.73 & $0.0040(-0.0458,0.0538)$ & 0.88 \\
\hline
\end{tabular}

Data are shown as $\beta(95 \% \mathrm{CI})$.

${ }^{1}$ This model did not control any confounding variable.

${ }^{2}$ Model I was adjusted for age, sex, ethnicity, education, PIR, CAD history, physical activity, obesity and smoking status.

${ }^{3}$ Model II was further adjusted for serum laboratory examinations, including total cholesterol, CRP, cadmium, folate, gamma tocopherol and vitamin

$\mathrm{A} / \mathrm{E} / \mathrm{B} 12$.

${ }^{4}$ Model III was further adjusted for dietary variables, including alcohol, caffeine, folate and vitamin B1/B2/B6/B12/C/E. 
Table 4. Coefficients and $95 \%$ CIs for peripheral leukocyte telomere length(bp) of vitamin A and carotenoid stratified for obesity.

\begin{tabular}{ccccc}
\hline & \multicolumn{1}{c}{ Vitamin A } & Carotenoid \\
\hline Total & $\mathrm{N}$ & $\beta(95 \% \mathrm{CI})$ & $\mathrm{N}$ & $\beta(95 \% \mathrm{CI})$ \\
Obesity & 839 & $0.0009(-0.0041,0.0058)$ & 839 & $0.0040(-0.0458,0.0538)$ \\
0 & & & 259 & $-0.1369(-0.2809,0.0070)$ \\
1 & 259 & $-0.0121(-0.0259,0.0017)$ & 580 & $0.0008(-0.0522,0.0539)$ \\
$P$ for interaction & 580 & $0.0003(-0.0050,0.0056)$ & & 0.04 \\
\hline
\end{tabular}

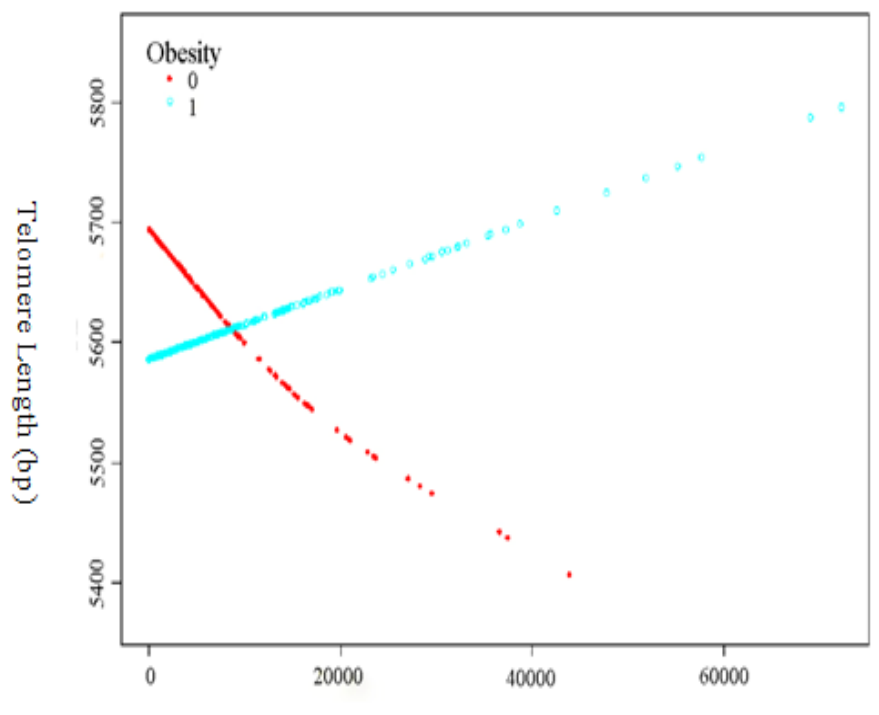

A

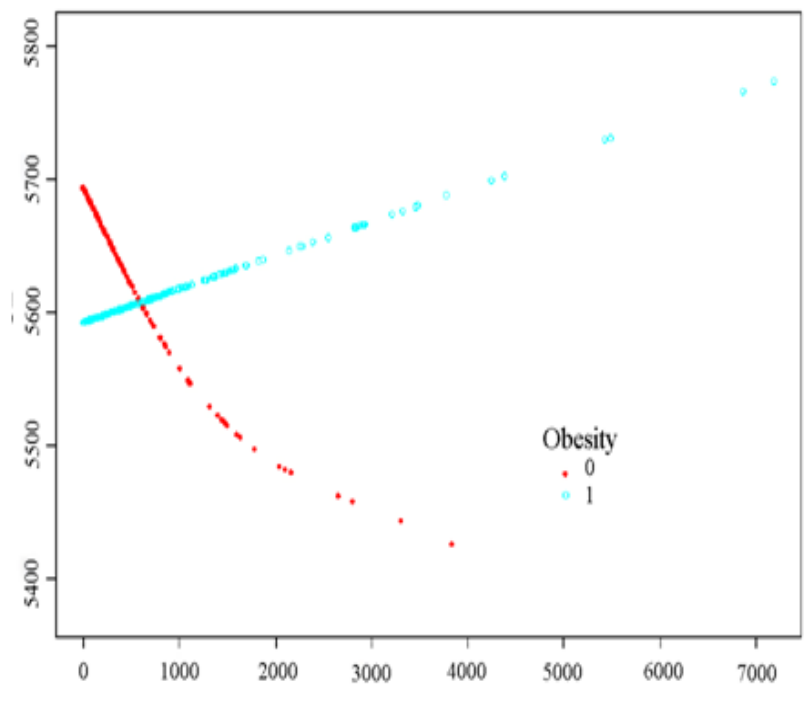

B

Figure 2. The association between peripheral telomere length and vitamin A(A) and Carotenoid(B). Adjusting for age, sex, ethnicity, education, PIR, CAD history, physical activity, smoking status, total cholesterol, CRP, cadmium, folate, gamma tocopherol, vitamin A/E/B12, alcohol consumption as well as dietary caffeine, folate and vitamin B1/B2/B6/B12/C/E. The red line standed for non-obesity, while the green one for obesity

However, for obesity individuals, the association of peripheral leukocyte telomere length with dietary vitamin A was $0.0003(95 \%$ CI: $-0.0050,0.0056)$, with dietary carotenoid was 0.0008 (95\% CI: $-0.0522,0.0539)$. Thus, dietary vitamin A or carotenoid intake was not significantly associated with the peripheral leukocyte telomere length.

Figure 2 showed the curve fitting association of peripheral telomere length (bp) with dietary vitamin A and carotenoid stratified with obesity. There was no turning point or platform stage in the curve, indicating no threshold saturation in the association between dietary vitamin A, carotenoid and telomere length.

\section{Conclusion}

There was no independent association between peripheral leukocyte telomere length and the dietary vitamin A or carotenoid in general population, adjusting confounding variant including age, race, sex, education, obesity, physical activity, PIR, smoking status, CAD history, serum concentrations of vitamin E/A/B12, folate, gamma-tocopherol, CRP, total cholesterol, cadmium as well as dietary intake of alcohol, caffeine, fiber, folate and dietary vitamin A/C/E/B1/B2/B6/B12. However, in non-obesity population, the dietary increase of vitamin A and carotenoid tended to associate with shorten peripheral leukocyte telomere length. Increasing intake of vitamin $\mathrm{A}$ and carotenoid in non-obese individuals might not benefit the length of peripheral leukocyte telomere length.

\section{Abbreviations}

Coronary artery disease (CAD), C-reactive protein (CRP), National Health and Nutrition Examination Survey (NHANES), poverty to income ratio (PIR), Metabolic Equivalents (METs), The National Institutes of Health (NIH).

\section{Funding}

This study was supported by National Natural Science Foundation of China (grant number: 81660210), Shaanxi Key research and development Program (grant number: 2018SF-107) and Xi'an Major Scientific and Technological Research Projects (grant number: 201805104YX12SF38(2)).

\section{Conflicts of Interest}

No conflict of interest was declared by authors. 


\section{References}

[1] Blackburn, E.H., "Structure and function of telomeres", Nature, 350(6319). 569-73. Sep. 1991.

[2] Blackburn, E.H., "Telomeres and telomerase: their mechanisms of action and the effects of altering their functions", FEBS Lett, 579(4). 859-62. Feb. 2005.

[3] Blasco, M.A., "Telomeres and human disease: ageing, cancer and beyond", Nat Rev Genet, 6(8). 611-22. Aug. 2005.

[4] Aubert, G. and P.M. Lansdorp, "Telomeres and aging", Physiol Rev, 88(2), 557-79. Apr. 2008.

[5] Fitzpatrick, A.L., et al., "Leukocyte telomere length and cardiovascular disease in the cardiovascular health study", $\mathrm{Am} \mathrm{J}$ Epidemiol, 165(1). 14-21. Jan. 2007.

[6] Brouilette, S.W., et al., "Telomere length, risk of coronary heart disease, and statin treatment in the West of Scotland Primary Prevention Study: a nested case-control study", Lancet, 369(9556). 107-14. Jan. 2007.

[7] Nowak, R., et al., "Telomere shortening and atherosclerosis", Lancet, 359(9310). 976-7. Mar. 2002.

[8] Willeit, P., et al., "Telomere length and risk of incident cancer and cancer mortality", JAMA, 304(1). 69-75. Jul. 2010.

[9] Cawthon, R.M., et al., "Association between telomere length in blood and mortality in people aged 60 years or older", Lancet, 361(9355). 393-5. Feb. 2003.

[10] Rode, L., B.G. Nordestgaard and S.E. Bojesen, "Peripheral blood leukocyte telomere length and mortality among 64,637 individuals from the general population", J Natl Cancer Inst, 107(6). djv074. Apr. 2015.

[11] Latifovic, L., et al., "The Influence of Alcohol Consumption, Cigarette Smoking, and Physical Activity on Leukocyte Telomere Length", Cancer Epidemiol Biomarkers Prev, 25(2). 374-80. Feb. 2016.

[12] Soares-Miranda, L., et al., "Physical Activity, Physical Fitness, and Leukocyte Telomere Length: The Cardiovascular Health Study", Med Sci Sports Exerc, 47(12). 2525-34. Dec. 2015.

[13] Mundstock, E., et al., "Effects of physical activity in telomere length: Systematic review and meta-analysis", Ageing Res Rev, 22. 72-80. Jul. 2015.

[14] Mundstock, E., et al., "Effect of obesity on telomere length: Systematic review and meta-analysis", Obesity (Silver Spring), 23(11). 2165-74. Nov. 2015.

[15] Mathur, M.B., et al., "Perceived stress and telomere length: A systematic review, meta-analysis, and methodologic considerations for advancing the field", Brain Behav Immun, 54. 158-169. May. 2016.

[16] Tucker, L.A., "Caffeine consumption and telomere length in men and women of the National Health and Nutrition Examination Survey (NHANES)", Nutr Metab (Lond), 14. 10. Jan. 2017.

[17] Needham, B.L., et al., "Socioeconomic status, health behavior, and leukocyte telomere length in the National Health and Nutrition Examination Survey, 1999-2002". Soc Sci Med, 85. 1-8. May. 2013.

[18] Cassidy, A., et al., "Associations between diet, lifestyle factors, and telomere length in women", Am J Clin Nutr, 91(5). 1273-80. May. 2010.

[19] Lee, J.Y., et al., Association between dietary patterns in the remote past and telomere length. Eur J Clin Nutr, 2015. 69(9): p. 1048-52.

[20] Tiainen, A.M., et al., "Leukocyte telomere length and its relation to food and nutrient intake in an elderly population", Er J Clin Nutr, 66(12). 1290-4. Dec. 2012.

[21] Tucker, L.A., "Consumption of Nuts and Seeds and Telomere Length in 5,582 Men and Women of the National Health and Nutrition Examination Survey (NHANES)", J Nutr Health Aging, 21(3). 233-240. 2017.

[22] Gu, Y., et al., "Mediterranean diet and leukocyte telomere length in a multi-ethnic elderly population", Age (Dordr), 37(2). 24. 2015.

[23] Hou, L., et al., "Telomere length in peripheral leukocyte DNA and gastric cancer risk", Cancer Epidemiol Biomarkers Prev, 18(11). 3103-9. Nov. 2009.

[24] Marcon, F., et al., "Diet-related telomere shortening and chromosome stability", Mutagenesis, 27(1). 49-57. Jan. 2012.
[25] Nettleton, J.A., et al., "Dietary patterns, food groups, and telomere length in the Multi-Ethnic Study of Atherosclerosis (MESA)", $\mathrm{Am}$ J Clin Nutr, 88(5). 1405-12. Nov. 2008.

[26] Leung, C.W., et al., "Soda and cell aging: associations between sugar-sweetened beverage consumption and leukocyte telomere length in healthy adults from the National Health and Nutrition Examination Surveys", Am J Public Health, 104(12). 2425-31. Dec. 2014.

[27] Von Zglinicki, T., "Oxidative stress shortens telomeres", Trends Biochem Sci, 27(7). 339-44. Jul. 2002.

[28] Lorenz, M., et al., "BJ fibroblasts display high antioxidant capacity and slow telomere shortening independent of hTERT transfection", Free Radic Biol Med, 31(6). 824-31. Sep. 2001.

[29] Zhang, D.H., et al., "DNA methylation of human telomerase reverse transcriptase associated with leukocyte telomere length shortening in hyperhomocysteinemia-type hypertension in humans and in a rat model", Circ J, 78(8). 1915-23. 2014.

[30] Richards, J.B., et al., "Homocysteine levels and leukocyte telomere length", Atherosclerosis, 200(2). 271-7. Oct. 2008.

[31] Tanumihardjo, S.A., "Vitamin A: biomarkers of nutrition for development", Am J Clin Nutr, 94(2). 658S-65S. Aug. 2011.

[32] McGrane, M.M., "Vitamin A regulation of gene expression: molecular mechanism of a prototype gene", J Nutr Biochem, 18(8) 497-508. Aug. 2007.

[33] Noy, N., "Between death and survival: retinoic acid in regulation of apoptosis". Annu Rev Nutr, 30. 201-17. Aug. 2010.

[34] Uray, I.P., E. Dmitrovsky and P.H. Brown, "Retinoids and rexinoids in cancer prevention: from laboratory to clinic", Semin Oncol, 43(1). 49-64. Sep. 2016.

[35] Licastro, F., et al., "Innate immunity and inflammation in ageing: a key for understanding age-related diseases". Immun Ageing, 2. 8. May. 2005.

[36] Reifen, R., "Vitamin A as an anti-inflammatory agent", Proc Nutr Soc, 61(3). 397-400. Aug. 2002.

[37] Stephensen, C.B., "Vitamin A, infection, and immune function", Annu Rev Nutr, 21. 167-92. 2001.

[38] Cooper, D.A., "Carotenoids in health and disease: recent scientific evaluations, research recommendations and the consumer", J Nutr, 134(1). 221S-224S. Jan. 2004.

[39] Krinsky, N.I. and E.J. Johnson, "Carotenoid actions and their relation to health and disease", Mol Aspects Med, 26(6). 459-516. Dec. 2005.

[40] Stahl, W. and H. Sies, "Bioactivity and protective effects of natural carotenoids", Biochim Biophys Acta, 1740(2). 101-7. May. 2005.

[41] Nomura, S.J., K. Robien and A.R. Zota, "Serum Folate, Vitamin B-12, Vitamin A, gamma-Tocopherol, alpha-Tocopherol, and Carotenoids Do Not Modify Associations between Cadmium Exposure and Leukocyte Telomere Length in the General US Adult Population", J Nutr, 147(4). 538-548. Apr. 2017.

[42] Min, K.B. and J.Y. Min, Association between leukocyte telomere length and serum carotenoid in US adults. Eur J Nutr, 56(3). 10451052. Apr. 2017.

[43] Mazidi, M., A.P. Kengne and M. Banach, "Mineral and vitamin consumption and telomere length among adults in the United States", Pol Arch Intern Med, 127(2). 87-90. Feb. 2017.

[44] CDC., U.S., NHANES 1999 - 2000 overview. Available at: https://wwwn.cdc.gov/nchs/nhanes/continuousnhanes/overview.as px?BeginYear=1999.

[45] Cawthon, R.M., "Telomere measurement by quantitative PCR", Nucleic Acids Res, 30(10). e47. May. 2002.

[46] Lin, J., et al., "Analyses and comparisons of telomerase activity and telomere length in human $\mathrm{T}$ and $\mathrm{B}$ cells: insights for epidemiology of telomere maintenance", $J$ Immunol Methods, 352(1-2). 71-80. Jan. 2010.

[47] CDC., U.S., NHANES 1999-2000 Data Documentation, Codebook, and Frequencies, Available at: https://wwwn.cdc.gov/Nchs/Nhanes/1999-2000/TELO_A.htm.

[48] CDC., U.S., NHANES 1999-2000 Data Documentation, Codebook, and Frequencies, Available at: https://wwwn.cdc.gov/Nchs/Nhanes/1999-2000/DRXTOT.htm A.htm.

[49] CDC., U.S., NHANES 1999 - 2000 Data Documentation, Codebook, and Frequencies, Available at: https://wwwn.cdc.gov/Nchs/Nhanes/1999-2000/MCQ.htm. 
[50] CDC., U.S., NHANES 1999 - 2000 Examination and Laboratory Procedures, Available at:

https://wwwn.cdc.gov/nchs/nhanes/continuousnhanes/manuals.asp $\mathrm{x}$ ?BeginYear $=1999$.

[51] CDC., U.S., National Health and Nutrition Examination SurveySurvey methods and analytic guidelines, Available at: https://wwwn.cdc.gov/nchs/nhanes/analyticguidelines.aspx..
[52] Rhinn, M. and P. Dolle, "Retinoic acid signalling during development", Development, 139(5). 843-58. Mar. 2012.

[53] Yabuzaki, J., "Carotenoids Database: structures, chemical fingerprints and distribution among organisms", Database (Oxford), 2017(1). Jan. 2017.

[54] NIH office of dietary supplements, Available at https://ods.od.nih.gov/factsheets/VitaminA-Consumer/.

(C) The Author(s) 2019. This article is an open access article distributed under the terms and conditions of the Creative Commons Attribution (CC BY) license (http://creativecommons.org/licenses/by/4.0/). 